\section{Recognition/confusion of dot matrix vs. conventional font capital letters}

\author{
L. H. GEYER and S. M. GUPTA \\ Department of Industrial Engineering \\ and Information Systems
}

Northeastern University, Boston, Massachusetts 02115

An earlier note (Gupta \& Geyer, 1980) discussed the apparent differences in confusion matrices obtained by Gilmore, Hersh, Caramazza, and Griffin (1979), using a video presentation of 5 by 7 dotmatrix capital letters, and by Townsend (1971a, 1971b), using tachistoscopic presentation of IBM Executive Diretrix capital letters. Such differences, if reliable and stable, would be of both theoretical and practical interest. From a theoretical viewpoint, comparisons among particularly divergent confusion cells for different fonts might illuminate the underlying attributes-features-commonly hypothesized to mediate recognition. From a practical viewpoint, improved recognition of video presentations of alphanumeric information could conceivably be obtained by using such comparisons to modify details of the dot matrix font.

However, it is difficult to feel complete confidence in the attribution of a major share of the differences between Gilmore et al.'s (1979) data and Townsend's (1971a, 1971b) to font differences. Presentation (tachistoscopic vs. video) and subjects provide plausible alternative sources of difference. The study summarized herein was designed to pinpoint the variation in font as a source of systematic differences in recognition/confusion.

A full 26 by 26 confusion matrix is too large to highlight font-specific differences. There are simply too many of the 676 cells that are virtually empty. This study used a selected set of nine letters as the stimulus set. All nine were rectangular rather than curved letters, and they were chosen to be two

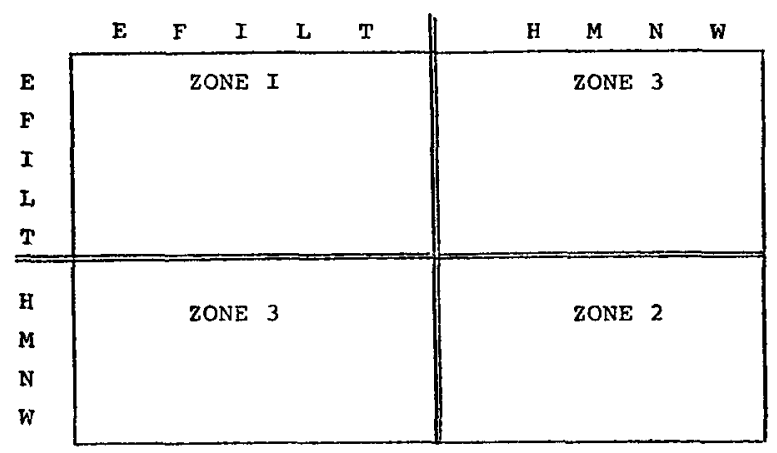

Figure 1. Confusion matrix partition lines defining zones.

\section{EFILTHHMH \\ E F I L T H M N W

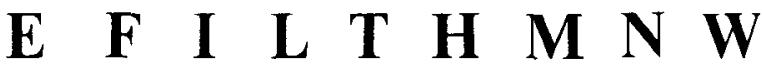

Figure 2. Nine stimulus letters in each of the three fonts.

specific subsets of five and four letters, respectively. The letters E, F, I, L, and T were selected because they were a group that had a substantial pattern of mutual confusion in Townsend's (1971a, 1971b) data and less mutual confusion in Gilmore et al.'s (1979) data. The letters $H, M, N$, and $W$ were selected as showing the opposite pattern. Furthermore, in these data sets there was not much confusion between the members of one subset with the members of the other.

Thereby, the confusion matrix for the selected nine letters can be conceptualized as shown in Figure 1, where there are three zones: Zone 1, comprising the recognition and confusion within subgroup for the letters $E, F, I, L$, and $T$; Zone 2, the same for the letters $H, M, N$, and $W$; and Zone 3, comprising the confusions of stimuli from one subgroup with responses from the other. Our hypothesis concerning font effects can be restated as follows: The pattern of confusion responses (i.e., responses off the main diagonal) for the three zones will be different for the different fonts: There will be more instances of above-average confusions in Zone 1 for a conventional "stroke" font than for the dot matrix font, and vice versa for Zone 2 .

So far we have discussed only two fonts. A third font was included in the study, specifically the font obtained by filling the spaces between the dots that were implicitly connected. This "filled" font was expected to be intermediate between dots and stroke. There was no specific hypothesis regarding comparative performance for the filled font. The nine stimulus letters in each of the three fonts used are shown in Figure 2.

A set of three confusion matrices, one for each font, were obtained in a series of experimental sessions, in each of which all stimulus letters in all fonts were presented equally often. Displays were presented such that the vertical angle subtended by each was $.55 \mathrm{deg}$, at a controlled energy level, and with duration intended to control correct recognition averaged across all fonts to .5 . 
Table 1

Aggregate Across-Subjects Confusion Matrices

\begin{tabular}{lccccccccc}
\hline & $\mathrm{E}$ & $\mathrm{F}$ & $\mathrm{I}$ & $\mathrm{L}$ & $\mathrm{T}$ & $\mathrm{H}$ & $\mathrm{M}$ & $\mathrm{N}$ & $\mathrm{W}$ \\
\hline & & & & \multicolumn{5}{c}{ Dots } \\
$\mathrm{E}$ & .40 & .18 & .04 & .14 & .05 & .08 & .01 & .05 & .06 \\
$\mathrm{~F}$ & .22 & .24 & .05 & .09 & .09 & .08 & .09 & .11 & .02 \\
$\mathrm{I}$ & .16 & .14 & .20 & .16 & .18 & .07 & .04 & .05 & .01 \\
$\mathrm{~L}$ & .20 & .09 & .08 & .39 & .03 & .07 & .04 & .06 & .04 \\
$\mathrm{~T}$ & .15 & .09 & .17 & .13 & .26 & .06 & .04 & .08 & .04 \\
$\mathrm{H}$ & .06 & .04 & .11 & .08 & .07 & .26 & .09 & .17 & .12 \\
$\mathrm{M}$ & .09 & .05 & .07 & .06 & .06 & .17 & .16 & .27 & .08 \\
$\mathrm{~N}$ & .04 & .04 & .05 & .06 & .04 & .16 & .09 & .42 & .11 \\
$\mathrm{~W}$ & .05 & .04 & .04 & .09 & .04 & .23 & .14 & .21 & .17 \\
& & & & & Filled & & & & \\
$\mathrm{E}$ & .83 & .08 & .01 & .05 & .01 & .00 & .01 & .01 & .01 \\
$\mathrm{~F}$ & .16 & .73 & .02 & .01 & .03 & .02 & .01 & .01 & .01 \\
$\mathrm{I}$ & .05 & .06 & .53 & .17 & .16 & .01 & .01 & .01 & .01 \\
$\mathrm{~L}$ & .09 & .01 & .05 & .77 & .02 & .02 & .00 & .03 & .02 \\
$\mathrm{~T}$ & .04 & .07 & .05 & .04 & .76 & .02 & .00 & .01 & .01 \\
$\mathrm{H}$ & .01 & .02 & .01 & .01 & .01 & .69 & .08 & .08 & .10 \\
$\mathrm{M}$ & .00 & .02 & .01 & .01 & .00 & .13 & .69 & .15 & .01 \\
$\mathrm{~N}$ & .02 & .00 & .00 & .00 & .01 & .03 & .04 & .88 & .03 \\
$\mathrm{~W}$ & .01 & .01 & .02 & .02 & .00 & .14 & .07 & .16 & .59 \\
& & & & & Stroke & & & & \\
$\mathrm{E}$ & .33 & .25 & .11 & .17 & .02 & .03 & .03 & .04 & .03 \\
$\mathrm{~F}$ & .13 & .50 & .24 & .05 & .04 & .02 & .01 & .01 & .01 \\
$\mathrm{I}$ & .06 & .11 & .68 & .08 & .06 & .00 & .00 & .00 & .00 \\
$\mathrm{~L}$ & .06 & .03 & .21 & .64 & .02 & .01 & .01 & .02 & .02 \\
$\mathrm{~T}$ & .07 & .10 & .32 & .06 & .42 & .01 & .00 & .02 & .00 \\
$\mathrm{H}$ & .00 & .00 & .00 & .00 & .00 & .69 & .13 & .14 & .05 \\
$\mathrm{M}$ & .00 & .00 & .01 & .00 & .00 & .01 & .91 & .03 & .05 \\
$\mathrm{~N}$ & .00 & .00 & .00 & .00 & .00 & .00 & .01 & .99 & .00 \\
$\mathrm{~W}$ & .00 & .00 & .00 & .00 & .01 & .00 & .01 & .00 & .98 \\
\hline & & & & & & & & &
\end{tabular}

The aggregated across-subjects confusion matrices for dot, filled, and stroke fonts are shown in Table 1. The mean correct response rate across all letters and fonts was . 55. The average correct response for dots was .28 , for filled .72 , and for strokes .68 . The difference in correct response due to font was significant $[F(2,24)=20.2, p<.001]$. The average dot recognition differed from that for filled $[\mathrm{t}(16)=$ $8.87, \mathrm{p}<.001]$ and from that for strokes $[\mathrm{t}(16)=$ $4.65, \mathrm{p}<.001]$. Average correct recognition performance for stroke and filled did not differ significantly $[\mathrm{t}(16)=.42]$.

The pattern of confusion errors, that is, the summation of the actual number of responses in the offdiagonal cells in Zones 1 and 2 and all cells in Zone 3 of Figure 1, is shown in Table 2. The chi-square values for comparison of confusion patterns between each pair of fonts are shown in Table 3 . These results support the hypothesis of differences in confusion patterns between dot and stroke fonts such that Zone 1 is larger for strokes than for dots and Zone 2 is larger for dots than for strokes.

From Table 2, it is clear that there is extremely little difference in confusion patterns for the comparison of dots and filled, a fact that is reflected in the chi-square value of .1 in Table 3 . In other words, whatever the basis for difference in confusion patterns between dots and stroke, it could not be solely due to the difference in overall recognition accuracy, because the same pattern of differences applies to the comparison of filled and stroke, when the average recognition levels did not differ. Indeed, the similarity in confusion patterns between dots and filled appears to be the most striking aspect of these data.

The present data seem to support the differences in performance expected from the earlier examination of the data reported by Gilmore et al. (1979) and Townsend (1971a, 1971b). Since the present data were obtained by tachistoscopic presentation and Gilmore et al. (1979) used a video presentation, it is interesting to attempt to compare the two sets of data for dots, as contrasted with a parallel comparison of the present data for strokes with those of Townsend (1971a, 1971b), who also used tachistoscopic presentation. Unfortunately, there is no rigorous way to compare a 26 by 26 matrix with a 9 by 9 one. As a qualitative approximation, we extracted the relevant 9 by 9 cells from the 26 by 26 matrix. Table 4 reports the count per zone of cells where the confusion datum exceeded an expected-by-chance value by a substantial margin. Table 4 gives the impression that the Townsend (1971a, 1971b) data and the strokes data match remarkably well, while the dots data do not conform to the Gilmore et al. (1979) data very well. There are several possible bases for this speculative finding (e.g., different sub-

Table 2

Pattern of Confusion Errors

\begin{tabular}{|c|c|c|c|c|c|c|}
\hline \multirow[b]{2}{*}{ Font } & \multicolumn{2}{|c|}{ Zone 1} & \multicolumn{2}{|c|}{ Zone 2} & \multicolumn{2}{|c|}{ Zone 3} \\
\hline & $\mathbf{S}$ & PE & $\mathbf{S}$ & $\mathrm{PE}$ & $\mathbf{S}$ & PE \\
\hline Dots & 411 & 33 & 228 & 18 & 613 & 49 \\
\hline Filled & 159 & 33 & 92 & 19 & 236 & 48 \\
\hline Stroke & 300 & 55 & 23 & 4 & 226 & 41 \\
\hline
\end{tabular}

Note-S = summation of off-diagonal cells; $P E=$ percentage of total errors.

Table 3

Chi-Square Values for Confusion Errors

\begin{tabular}{lcc}
\hline & Filled & Stroke \\
\hline Dots & .1 & 104.8 \\
Filled & & $\mathbf{8 1 . 5}$ \\
\hline
\end{tabular}

Table 4

Number of Cells That Exceed Chance

\begin{tabular}{llcc}
\hline & \multicolumn{3}{c}{ Zone } \\
\cline { 2 - 4 } & 1 & 2 & 3 \\
\hline Townsend & 9 & 2 & 0 \\
Stroke & 9 & 2 & 0 \\
Dots & 5 & 5 & 0 \\
Gilmore et al. & 2 & 10 & 0 \\
\hline
\end{tabular}


jects), but one that might be of interest, if it were shown to be true, is that there are some systematic differences attributable to the difference between tachistoscopic and video stimulation.

\section{REFERENCES}

Gilmore, G. C., Hersh, H., Caramazza, A., \& Griffin, J. Multidimensional letter similarity derived from recognition errors. Perception \& Psychophysics, 1979, 25, 425-431.
Gupta, S. M., \& Geyer, L, H. On tactile and visual recognition. Perception \& Psychophysics, 1980, 27, 579-580.

Townsend, J. T. Alphabetic confusion: A test of models for individuals. Perception \& Psychophysics, 1971, 9, 449-454. (a) Townsend, J. T. Theoretical analysis of an alphabetic confusion matrix. Perception \& Psychophysics, 1971, 9, 40-50. (b)

(Received for publication February 2, 1981; accepted February 2, 1981.) 